


\title{
ECOGRAPHIC CHARACTERISTICS OF THE POST CAESAREAN SECTION SCAR IN THE LOWER UTERINE SEGMENT IN NON-PREGNANT PATIENTS
}

\section{CARACTERÍSTICAS ECOGRÁFICAS DE LA CICATRIZ POSTCESÁREA EN EL SEGMENTO UTERINO INFERIOR EN PACIENTES NO GESTANTES}

\author{
Nataly Paola Negrette Lopera* \\ Jackeline Jaimes Becerra**
}

* Autor principal: Médico especialista en Ginecología y Obstetricia, Departamento de Ginecología y Obstetricia, Escuela de Medicina, Universidad Industrial de Santander (UIS), Bucaramanga, Colombia. Dirección de correspondencia: Carrera 32 № 29-31, Teléfono: (57) (7) 6324929 - 6343496, correo electrónico: natyntte@ hotmail.com

** Médico especialista en Ginecología y Obstetricia, Profesor Titular, Departamento de Ginecología y Obstetricia, Escuela de Medicina, Universidad Industrial de Santander, Bucaramanga, Colombia. Dirección de correspondencia: Carrera 32 № 29-31, Teléfono: (57) (7) 6324929 - 6343496, correo electrónico: jaimesjackeline@ gmail.com

Summary: With the increase in the rate of caesarean sections, more women experience longterm effects, because more than half of them develop a defect in the scar after a cesarean section. These defects are related to abnormal bleeding, dysmenorrhea, chronic pelvic pain, subfertility and obstetric problems. It has been demonstrated that transvaginal ultrasound is an accurate method to evaluate the uterine scar after cesarean and the presence of defects, which can predict the risk of complications derived from it. We decided to evaluate, by transvaginal ultrasound, the characteristics of the post-cesarean scar in the lower uterine segment in non-pregnant patients. We conducted a descriptive study with prospective collection in this population and reported the outcome in 140 patients, between 18 and 45 
years of age, with a history of cesarean section. We identified 126 scars and 10 patients with double scars by transvaginal ultrasound. One hundred and seventeen scars $(87.5 \%)$ had a residual myometrium $>2.2 \mathrm{~mm}$. The prevalence of defects was $73.1 \%, 57(41.9 \%)$ had a mild defect, $67(49.2 \%)$ moderate and $12(8.9 \%)$ severe. Four factors were associated with the presence of defect in the scar: more than one cesarean (OR 2.43, 95\% CI 1.07-5.55), elective cesarean (OR 4.79, 95\% CI 1.93-12, 9), more than one scar and the distance from the scar to the OCI (OR 0.77 IC95\% 0.663-0.90). We did not show statistical association with the position of the uterus, with gynecological symptoms or with the demographic characteristics of the patients. These results are similar to those reported in the literature. The distance of the scar to the OCI is a protective factor for the formation of defects as elective caesarean section is associated with anomalous scars.

Resumen: Con el aumento de la tasa de cesáreas, más mujeres experimentan efectos a largo plazo, debido a que más de la mitad desarrollan un defecto en la cicatriz después de una cesárea. Estos defectos están relacionados con sangrado anormal, dismenorrea, dolor pélvico crónico, subfertilidad y problemas obstétricos. Se ha evidenciado que la ecografía transvaginal es un método preciso para evaluar la cicatriz uterina posterior a cesárea y la presencia de defectos, la cual puede predecir el riesgo de complicaciones derivadas de esta. Decidimos evaluar por ecografía transvaginal las características de la cicatriz postcesárea en el segmento uterino inferior en pacientes no embarazadas. Realizamos un estudio descriptivo con recolección prospectiva en esta población e informamos el resultado en 140 pacientes, entre los 18 y 45 años de edad, con antecedente de cesárea. Identificamos por ecografía transvaginal 126 cicatrices y 10 pacientes con doble cicatriz. Ciento diecisiete cicatrices $(87,5 \%)$ tenían un miometrio residual $>2,2 \mathrm{~mm}$. La prevalencia de defectos fue del 73,1\%, $57(41,9 \%)$ tenían un defecto leve, 67 (49,2\%) moderado y 12 (8,9\%) severo. Cuatro factores se asociaron con la presencia de defecto en la cicatriz: más de una cesárea (OR 2,43, IC95\% 1,07-5,55), cesárea electiva (OR 4,79, IC95\% 1,93-12,9), más de una cicatriz y la distancia de la cicatriz al OCI (OR 0,77 IC95\% 0,663-0,90). No evidenciamos asociación estadística con la posición del útero, con síntomas ginecológicos o con las características demográficas de las pacientes. Estos resultados son similares a los reportados en la literatura. La distancia de la cicatriz al OCI es un factor protector para la formación de defectos así como la cesárea electiva se asocia con cicatrices anómalas. 


\section{ABSTRACT}

Background: with the increase in the rate of caesarean sections, more women experience long-term effects, because more than half develop a defect in the scar after a cesarean section. These defects are related to abnormal bleeding, dysmenorrhea, chronic pelvic pain, subfertility and obstetric problems.

Objective: evaluate by transvaginal ultrasound the post-caesarean scar in the lower uterine segment in non-pregnant patients and explore the possible factors involved in the healing of the caesarean, the development of scar defects and their association with gynecological symptoms.

Study Design: descriptive study, with prospective collection. There were 15 questions about obstetric history and gynecological symptoms. Transvaginal ultrasound was performed on the participants and the following measures were taken: length, width and depth of the scar, residual myometrium (RM) and distance of the scar to the internal cervical os (ICO). If they presented scar defects, the appearance of the scar was described.

Results: 140 patients were evaluated, between 18 and 45 years old with antecedent of cesarean section. 126 scars (90\%) were identified sonographically, 10 participants (7,9\%) had double scars and 98 scars $(73.1 \%)$ had some type of defect. Of the visible scars, 57 (4.9\%) had a mild defect, 67 (49.2\%) moderate and 12 (8.9\%) severe. One hundred and seventeen scars $(87.5 \%)$ had a RM $>2.2 \mathrm{~mm}$. Four factors presented association whit the presence of defect: more than one cesarean, effective cesarean, more than are scar and the distance of the scar to the internal cervical os (ICO). There was no statically significant relationship with the presence of defects and gynecological symptoms.

Conclusion: Our results are similar to those reported in the literature. The RM and the percentage of RM are maintained as important factors in determining the healing of a cesarean. The distance of the scar to the (ICO) a protective factor for the formation of defects, as the elective cesarean is associated with anomalous scars.

Key words: vaginal ultrasound, caesarean sections, scar, non-pregnant, gynecological symptoms. 


\section{RESUMEN}

Antecedentes: con el aumento de la tasa de cesáreas, más mujeres experimentan efectos a largo plazo, debido a que más de la mitad desarrollan un defecto en la cicatriz después de una cesárea. Estos defectos están relacionados con sangrado anormal, dismenorrea, dolor pélvico crónico, subfertilidad y problemas obstétricos.

Objetivo: evaluar ecográficamente la cicatriz postcesárea en el segmento uterino inferior en pacientes no embarazadas y explorar los posibles factores que intervienen en la cicatrización de la cesárea, el desarrollo de defectos en la cicatriz y su asociación con síntomas ginecológicos.

Diseño del estudio: estudio descriptivo, con recolección prospectiva. Se realizaron 15 preguntas sobre antecedentes obstétricos y síntomas ginecológicos. Se realizó ecografía transvaginal a las participantes y se tomaron las siguientes medidas: longitud, amplitud y profundidad de la cicatriz, miometrio residual (MR) y distancia de la cicatriz al orificio cervical interno (OCI). Si presentaban defectos en la cicatriz se describió la apariencia del mismo.

Resultados: se evaluaron 140 pacientes, entre los 18 y 45 años con antecedente de cesárea. Se identificaron ecográficamente 126 cicatrices (90,0\%), 10 participantes (7,9\%) tenían doble cicatriz y 98 cicatrices $(73,1 \%)$ presentaban algún tipo de defecto. De las cicatrices visibles, 57 (41,9\%) tenían un defecto leve, 67 (49,2\%) moderado y 12 (8,9\%) severo. Ciento diecisiete cicatrices $(87,5 \%)$ tenían un MR >2,2 mm. Cuatro factores presentaron asociación con la presencia de defecto: más de una cesárea, cesárea electiva, más de una cicatriz y la distancia de la cicatriz al OCI. No hubo relación estadísticamente significativa con la presencia de defectos y síntomas ginecológicos.

Conclusión: nuestros resultados, son similares a los reportados en la literatura. El MR y el porcentaje de MR se mantienen como factores importantes para determinar la cicatrización de una cesárea. La distancia de la cicatriz al OCI es un factor protector para la formación de defectos así como la cesárea electiva se asocia con cicatrices anómalas.

Palabras clave: ecografía transvaginal, cesárea, cicatriz, no embarazadas, síntomas ginecológicos. 


\section{INTRODUCCIÓN}

En las últimas décadas, las tasas de cesárea han aumentado en todo el mundo, tanto en países en desarrollo como en los países desarrollados. En Estados Unidos la incidencia de cesárea es del 26,1\%, en América Latina del 29,0\% en promedio, observándose los índices más elevados en México, Brasil, Colombia, República Dominicana y Chile, los cuales oscilan entre 30,0\% y 39,0\% (1). La Organización Mundial de la Salud (OMS) estima que la tasa ideal de cesáreas debe ser menor al 15,0\% (2), sin embrago, para el año 2017, en Colombia, la tasa de cesárea fue de 46,2\% y en Santander de 53,8\% (3), datos que superan lo recomendado por la OMS.

El aumento de las tasas de cesárea implica que las mujeres con una cicatriz de cesárea constituyen una población en rápido crecimiento, presentando riesgos a largo plazo derivados de dicha cicatriz como formación de adherencias pélvicas postoperatorias, dehiscencia y ruptura uterina, alteraciones en la placentación, presencia de embarazo ectópico, dolor pélvico crónico, sangrados uterinos anormales e infertilidad, además esos riesgos se elevan significativamente en caso de múltiples cesárea (4).

El hallazgo ecográfico observado en el área de la cicatriz uterina corresponde generalmente a un área anecóica, localizada en la unión útero-cervical anterior, que forma una solución de continuidad en el miometrio anterior, que se enfrenta a la cavidad endometrial (5).Varios grupos han considerado durante el seguimiento de pacientes intervenidas por cesárea que, la presencia de un defecto en la cicatriz uterina o una disminución del espesor del miometrio residual, son un marcador de mala cicatrización, por lo que la ecografía transvaginal es un método preciso para evaluar la cicatriz uterina posterior a cesárea y la presencia de defectos, permitiendo la posibilidad de predecir el riesgo de complicaciones derivadas de esta (6).

Debido a la relevancia de las implicaciones de una cicatriz en el útero y la presencia o no de defectos, es importante determinar los posibles factores que podrían desempeñar un papel importante en la cicatrización de la cesárea y el desarrollo de defectos en la cicatriz así como la identificación de esta ecográficamente (7). 


\section{DISEÑO DEL ESTUDIO}

Se realizó un estudio descriptivo, con recolección prospectiva, realizado entre Febrero y Diciembre de 2017 en pacientes que tenían indicación de realización de ecografía transvaginal que consultaron al servicio de ecografía ginecológica del Hospital Universitario de Santander, Colombia. Los criterios de inclusión fueron pacientes en edad reproductiva, no embarazadas, que tuvieran antecedente de cesárea. Se excluyeron pacientes que presentaran patologías uterinas que distorsionaran la morfología normal del útero, pacientes con cicatrices uterinas diferentes a cesárea segmentaria y mujeres dentro de los primeros 40 días postparto.

Las pacientes que cumplieran con los criterios de inclusión y que firmaron el consentimiento informado, se les realizó una encuesta de 15 preguntas sobre antecedentes obstétricos y síntomas ginecológicos. La ecografía transvaginal y la evaluación de la cicatriz fue realizada con ecógrafo GE Voluson E6 con transductor endovaginal de 7.5 MHz, por un operador con la paciente en decúbito supino y la vejiga desocupada. La cicatriz uterina se evaluó en ambos planos sagital y transversal y se evidenció entre el pliegue uterovesical y el miometrio. A aquellas cicatrices que eran visibles ecográficamente, se le tomaron las siguientes medidas: longitud, amplitud y profundidad de la cicatriz, miometrio residual y distancia de la cicatriz al orificio cervical interno. Si presentaban defectos en la cicatriz se describió la apariencia del mismo. El protocolo del estudio fue aprobado por el Comité Institucional de Ética Médica de la UIS.

\section{ANÁLISIS ESTADÍSTICO}

Inicialmente se realizó un análisis univariado para describir las características de la población estudiada y se detallaron las características ecográficas de la cicatriz uterina. Adicionalmente se realizó un análisis bivariado comparando las pacientes con defecto de la cicatriz con aquellas que no lo tenían, para finalmente generar un modelo de regresión logística; así se exploraron qué características de la paciente influyen en la formación del defecto. Se realizó comparación entre la presencia de defectos y la presencia de síntomas ginecológicos, se consideró un valor significativamente estadístico $p<0.05$. Los datos obtenidos se tabularon en una base de datos en Microsoft Excel ${ }^{\circledR}$, en donde a cada paciente 
se le asignó un código interno para identificarlas. El análisis estadístico se realizó en Stata 12.1 (StataCorp, College Station, EU, 2014).

\section{RESULTADOS}

Se recolectaron 140 pacientes con edad entre 18 y 45 años (media 32, recorrido intercuartil (RIQ) de 26,5 a 36,5). El peso de las pacientes estuvo entre 35 y $120 \mathrm{Kg}$ (media de 64,0, RIQ de 58,0 a 72,0) y la talla entre 1,41 y 1,72 Mts (media de 1,59, RIQ de 1,55 y 1,62); así el IMC osciló entre 17,6 y 44,5kg/m² (media de 25,0, RIQ de 23,4 y 27,2) (Tabla 1).

Tabla 1. Medidas antropométricas de la población estudiada a quienes se les realizó ecografía transvaginal

\begin{tabular}{|l|c|c|c|c|}
\hline \multicolumn{1}{|c|}{$\begin{array}{c}\text { MEDIDAS } \\
\text { ANTROPOMÉTRICAS }\end{array}$} & $\begin{array}{c}\text { VALOR } \\
\text { INFERIOR }\end{array}$ & $\begin{array}{c}\text { VALOR } \\
\text { SUPERIOR }\end{array}$ & MEDIA & RIQ \\
\hline Peso (Kg) & 35 & 120 & 64 & $58-72$ \\
\hline Talla (Mts) & 1,41 & 1,72 & 1,59 & $1,55-1,62$ \\
\hline IMC & 17,6 & 44,5 & 25,0 & $23,4-27,2$ \\
\hline
\end{tabular}

El número de gestaciones que habían presentado fue entre 1 y 7 . Cincuenta pacientes $(35,5 \%)$ tuvieron entre 1 y 5 partos, y $45(32,0 \%)$ entre 1 y 3 abortos; cinco pacientes $(3,6 \%)$ un embarazo ectópico, 2 (1,4\%) un mortinato y 2 (1,4\%) un embarazo gemelar (Tabla 2). 
Tabla 2. Historia obstétrica de las pacientes evaluadas

\begin{tabular}{|l|l|c|c|}
\hline VARIABLE & PACIENTES & \% \\
\hline \multirow{4}{*}{ Gestaciones } & 1 & 32 & 22,8 \\
\cline { 2 - 4 } & $2-4$ & 97 & 69,3 \\
\cline { 2 - 4 } & $5-7$ & 11 & 7,8 \\
\hline \multirow{3}{*}{ Partos Vaginales } & 0 & 90 & 64,3 \\
\cline { 2 - 4 } & $1-2$ & 41 & 33,6 \\
\cline { 2 - 4 } & $3-5$ & 9 & 2,1 \\
\hline \multirow{3}{*}{ Abortos } & 0 & 95 & 67,8 \\
\cline { 2 - 4 } & 1 & 40 & 28,5 \\
\cline { 2 - 4 } & $\geq 2$ & 5 & 3,5 \\
\hline \multirow{2}{*}{ Ectópicos } & 0 & 134 & 95,7 \\
\cline { 2 - 4 } & 1 & 6 & 4,3 \\
\hline \multirow{2}{*}{ Mortinatos } & 0 & 138 & 98,6 \\
\cline { 2 - 4 } & 1 & 138 & 1,4 \\
\cline { 2 - 4 } & 1 & 2 & 98,6 \\
\hline
\end{tabular}

El total de la población tenía entre 1 y 4 cesáreas (Figura 1), de las cuales 84 (60,0\%) fueron electivas (Figura 2). La edad gestacional al momento de la cesárea fue entre 32 y 41 semanas (media de 39, RIQ de 37,4 y 39) y el peso del recién nacido estuvo entre 1343 a 4200 gramos (media de 3200, RIQ de 2900 y 3500).

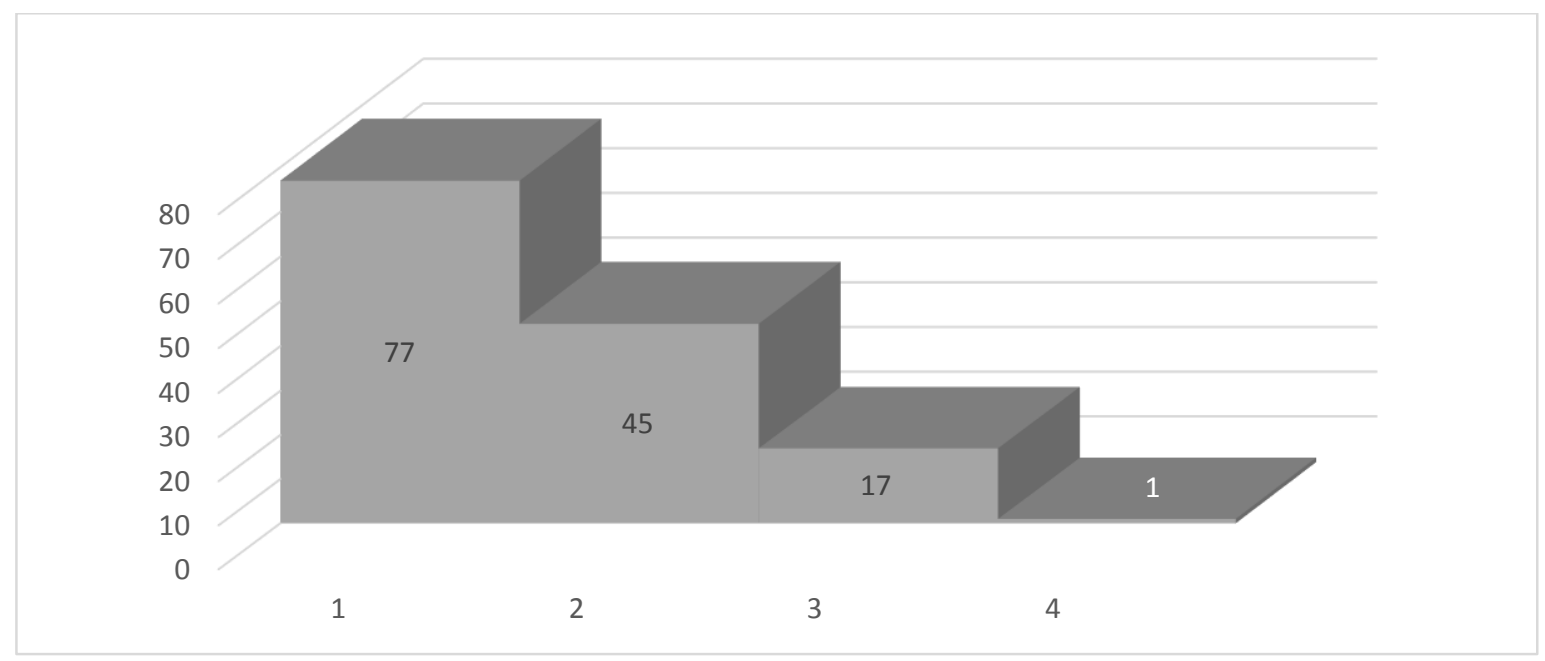

Figura 1. Número de cesáreas que habían presentado las pacientes hasta el momento de la ecografía. 




Figura 2. Tipo de cesárea realizadas a las pacientes evaluadas.

Sesenta y cuatro pacientes $(45,7 \%)$ tenían indicación de ecografía transvaginal por alteraciones en el ciclo y sangrado menstrual, 69 (49,2\%) por dolor y $7(5,1 \%)$ por infertilidad (Figura 3). Las alteraciones del ciclo y volumen menstrual así como el tipo de dolor referidos por las pacientes, se muestran en las figuras 4 y 5 .

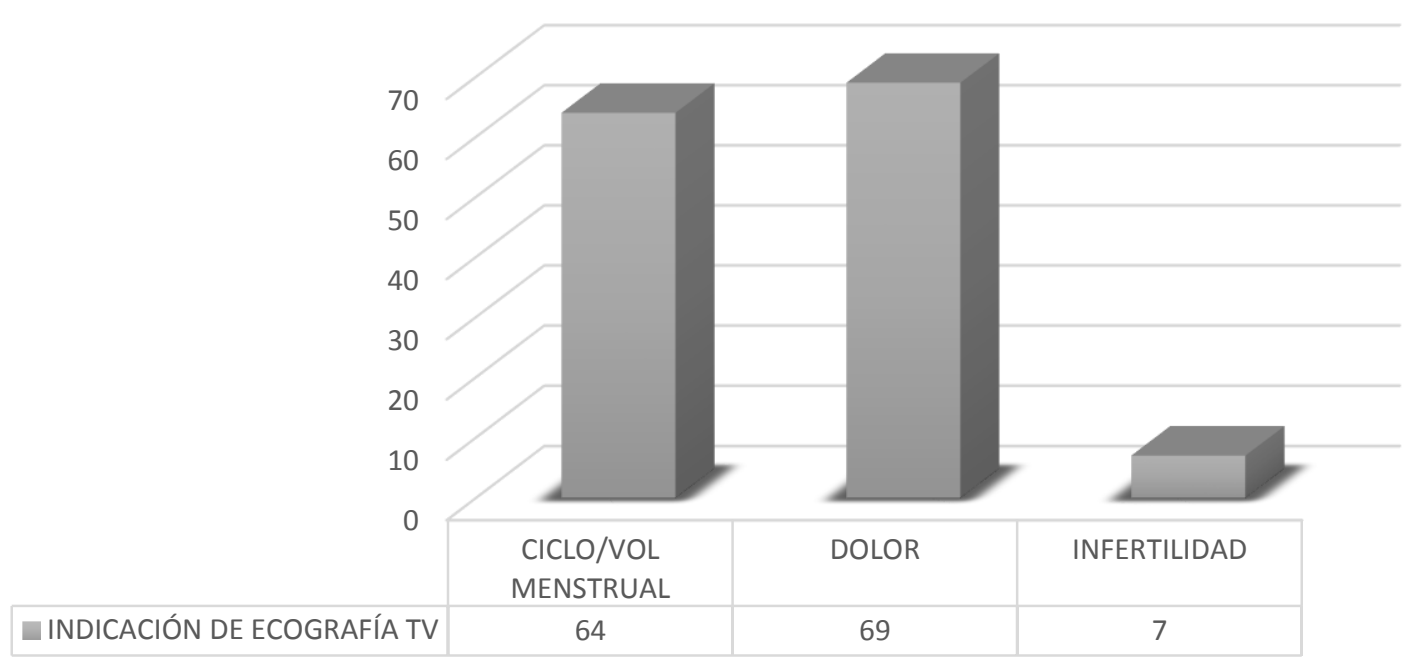

Figura 3. Motivo de realización de la ecografía transvaginal. 


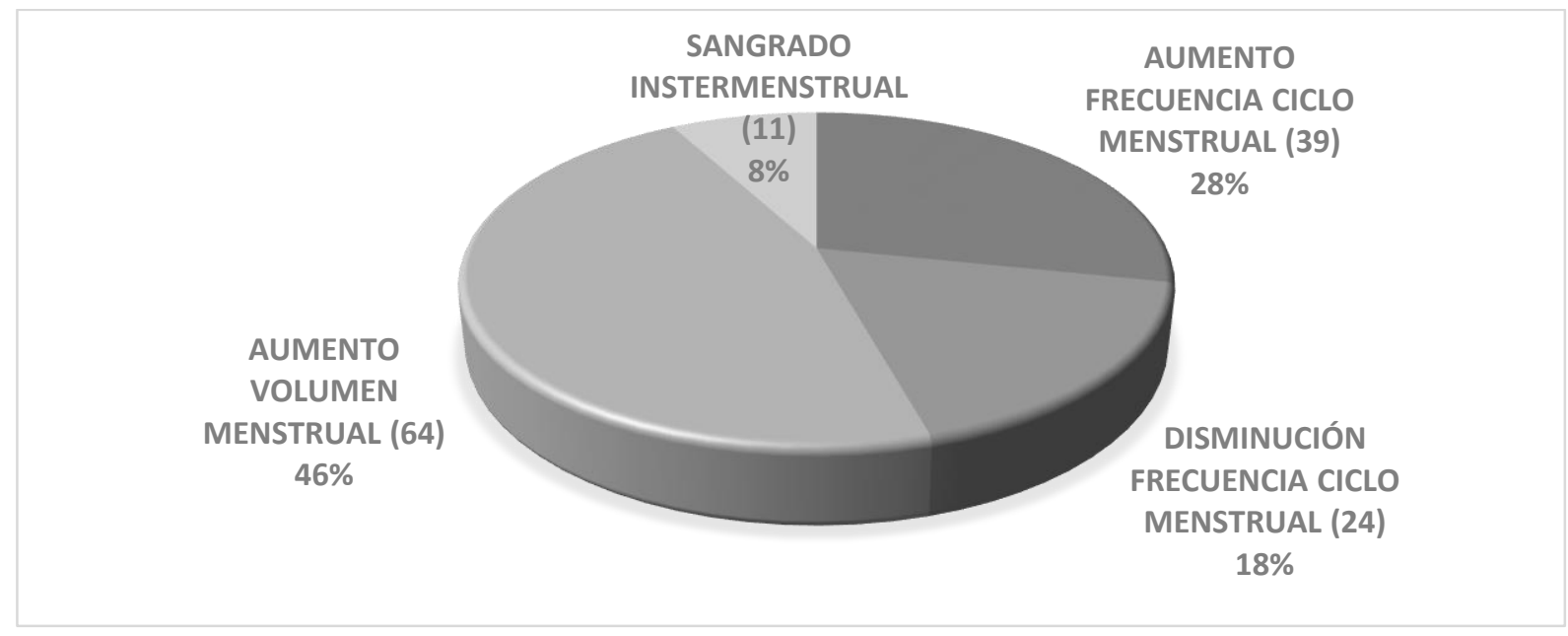

Figura 4. Síntomas manifestados por las pacientes evaluadas en relación con alteración del ciclo y volumen menstrual.

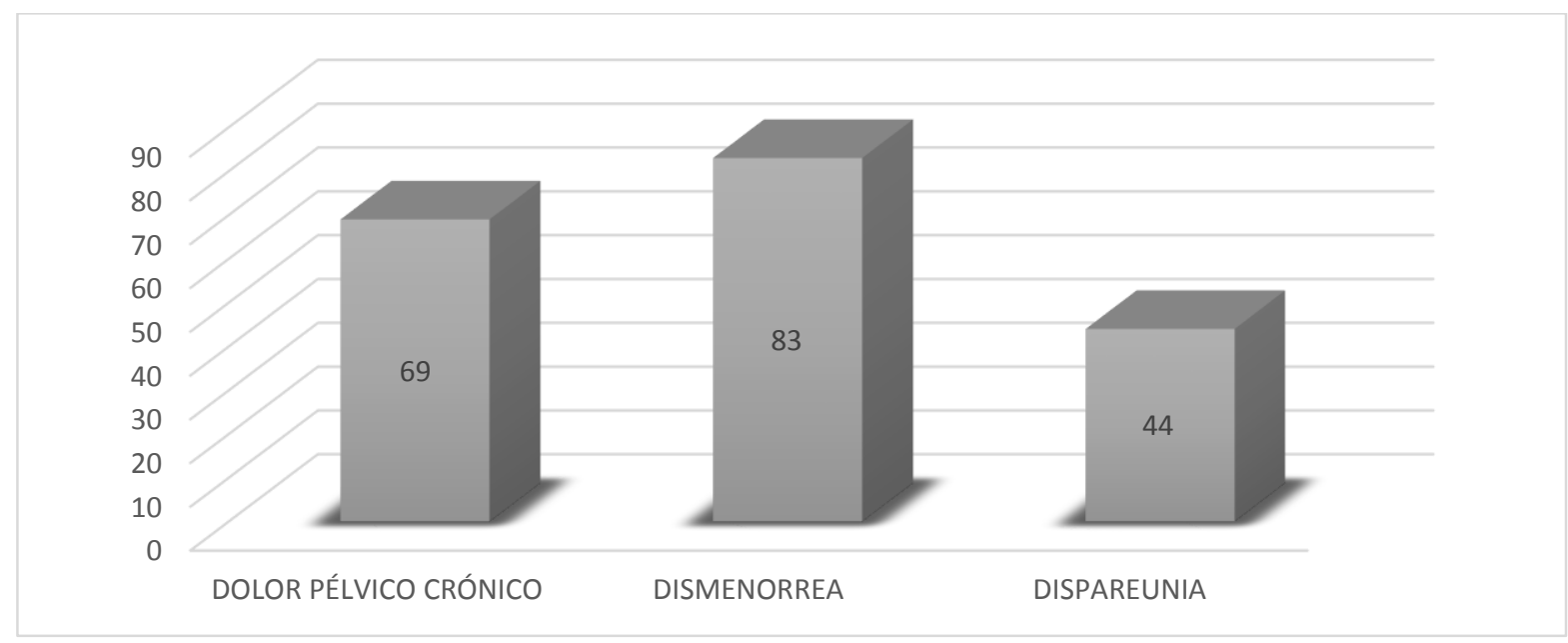

Figura 5. Síntomas manifestados por las pacientes evaluadas en relación con dolor.

El tiempo transcurrido entre la cesárea y la realización de la ecografía fue entre 1 y 20 años (media de 5, RIQ de 2 y 9). Un total de 110 pacientes $(78,5 \%)$ presentaron útero en AVF y $30(21,4 \%)$ en RVF. Ciento veintiséis cicatrices (90,0\%) se identificaron y 14 (10,0\%) no pudieron ser observadas. Diez pacientes $(7,9 \%)$ tenían dos cicatrices visibles (Figura 6). 


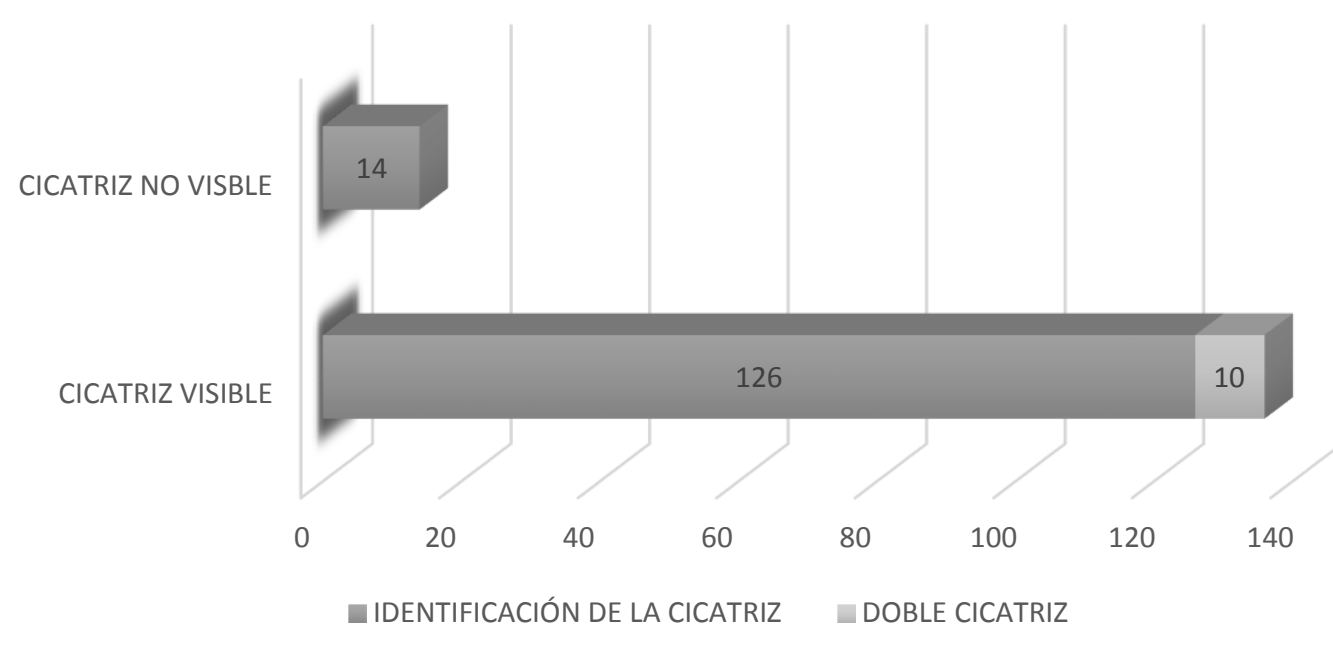

Figura 6. Identificación ecográfica de las cicatrices uterinas en las pacientes evaluadas.

Las diferentes medidas evaluadas ecográficamente de las cicatrices identificadas, se muestran en la Tabla 3.

Tabla 3. Variables ecográficas de las cicatrices visibles ecográficamente.

\begin{tabular}{|c|c|c|c|c|}
\hline $\begin{array}{c}\text { VARIABLE } \\
\text { ECOGRÁFICA }\end{array}$ & $\begin{array}{c}\text { VALOR } \\
\text { INFERIOR }\end{array}$ & $\begin{array}{c}\text { VALOR } \\
\text { SUPERIOR }\end{array}$ & MEDIA & RIQ \\
\hline Longitud (mm) & 1,3 & 13,2 & 5,9 & $4,4-7,6$ \\
\hline Amplitud (mm) & 1,3 & 14,3 & 4,6 & $2,7-6,8$ \\
\hline $\begin{array}{c}\text { Profundidad } \\
(\text { mm) }\end{array}$ & 1,6 & 15,4 & 4,9 & $3,3-7,1$ \\
\hline $\begin{array}{c}\text { Miometrio } \\
\text { residual (mm) }\end{array}$ & 0,9 & 10,0 & 4,1 & $2,7-5,4$ \\
\hline $\begin{array}{c}\text { \% Miometrio } \\
\text { residual }\end{array}$ & 12,8 & 82 & 45,5 & $32,0-57,7$ \\
\hline
\end{tabular}

De las cicatrices que fueron visibles, $98(73,1 \%)$ presentaban algún tipo de defecto reconocido ecográficamente (Figura 7). Según la puntuación de Tower y Frishman, del total 
de cicatrices visibles, 57 (41,9\%) tenían un defecto leve, 67 (49,2\%) moderado y 12 (8,9\%) severo (Tabla 4). Treinta y cinco de los defectos $(36,0 \%)$ tenia forma triangular, $31(32,0 \%)$ era de forma circular, $24(24,4 \%)$ forma de cajón y $8(8,1 \%)$ forma de quistes de inclusión (Figura 8).

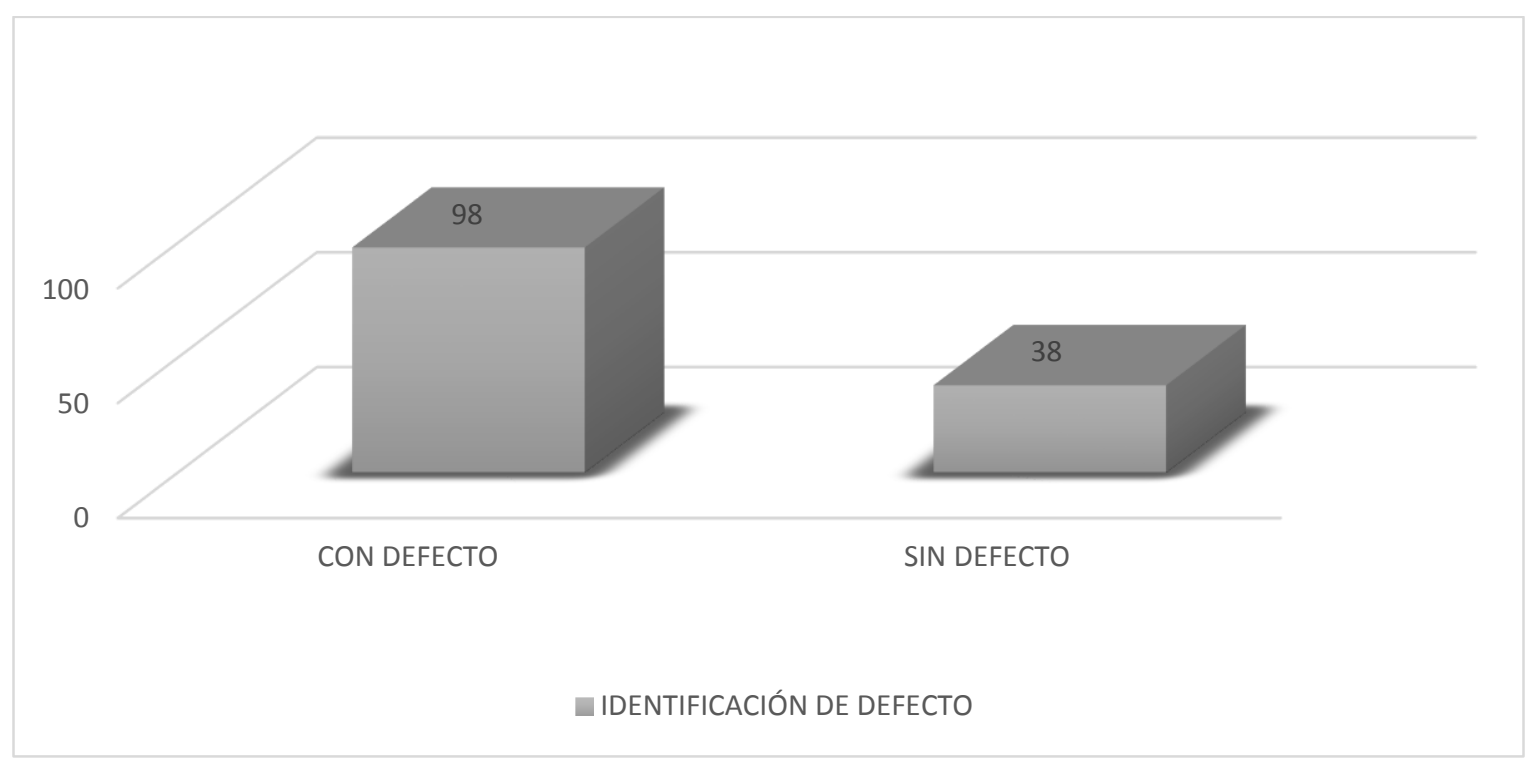

Figura 7. Identificación de defectos en las cicatrices uterinas.

Tabla 4. Tipo de defectos según puntuación de Tower y Frishman de las cicatrices observadas.

\begin{tabular}{|l|c|c|c|}
\hline \multirow{2}{*}{$\begin{array}{c}\text { TIPO DE } \\
\text { DEFETO DE LA } \\
\text { CICATRIZ }\end{array}$} & PUNTUACIÓN & CICATRICES & \% \\
\hline \multirow{2}{*}{ Leve } & & & \\
\hline \multirow{3}{*}{ Moderado } & 2 & 29 & 21,3 \\
\cline { 2 - 4 } & 3 & 28 & 20,6 \\
\hline \multirow{3}{*}{ Severo } & 4 & 32 & 23,5 \\
\cline { 2 - 4 } & 5 & 24 & 17,6 \\
\cline { 2 - 4 } & 6 & 11 & 8,1 \\
\cline { 2 - 4 } & 7 & 5 & 3,7 \\
\hline
\end{tabular}




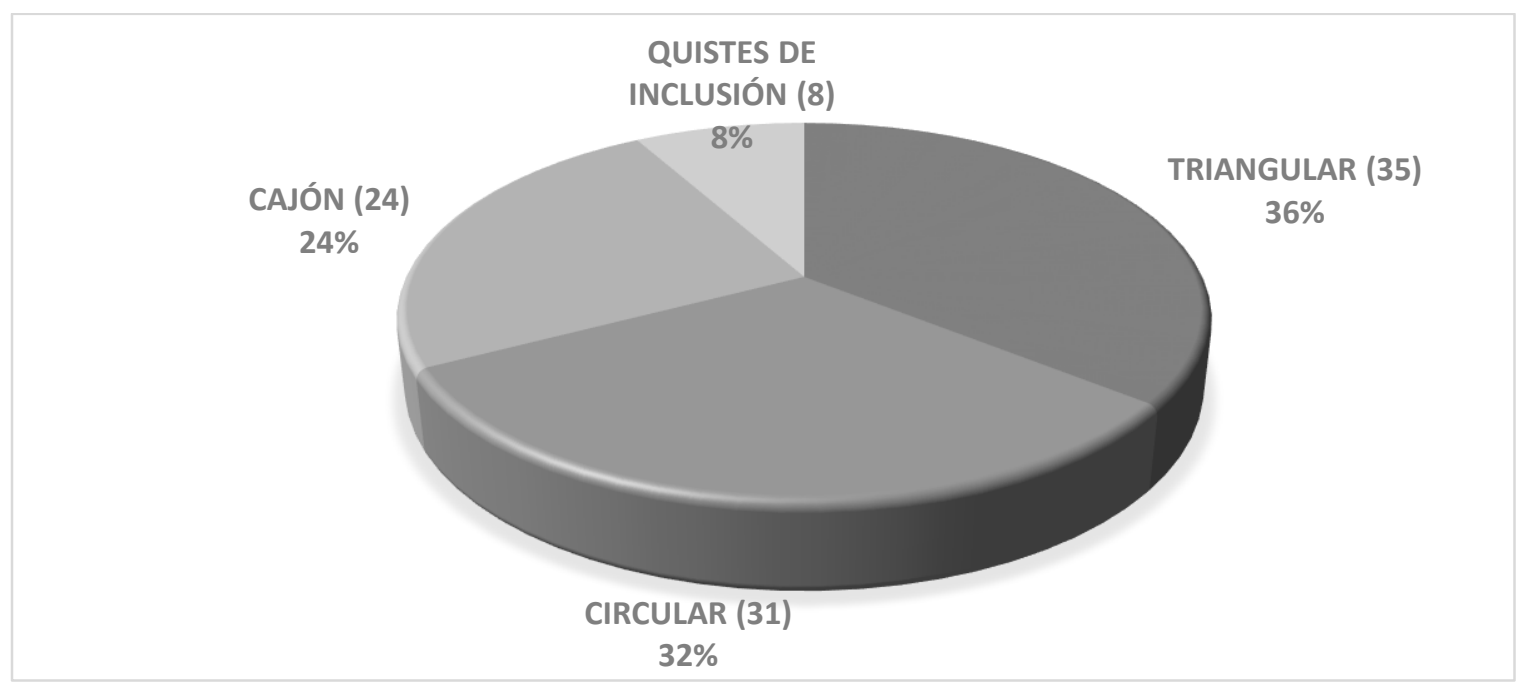

Figura 8. Forma del defecto en las cicatrices uterinas.

En el análisis bivariado se encontraron cuatro factores asociados con la presencia de defecto en la cicatriz: más de una cesárea (OR 2,43, IC95\% 1,07-5,55), cesárea electiva (OR 4,79, IC95\% 1,93-12,9), más de una cicatriz y la distancia de la cicatriz al OCI (OR 0,77 IC95\% 0,663-0,90). Sin embargo, en el modelo multivariado solamente dos de ellos están realmente asociados: la historia de cesárea electiva (OR 3.23, IC95\% 1.21-8.62) y la distancia de la distancia de la cicatriz al OCI (OR 0.792, IC95\% 0.666-0.941); el modelo de regresión logística ajusta adecuadamente ( $\mathrm{p}=0.390)$ (Tabla 5).

Tabla 5. Factores asociados al defecto de la cicatriz de la cesárea

\begin{tabular}{|c|c|c|c|}
\hline \multirow[b]{2}{*}{ CARACTERÍSTICAS } & \multicolumn{2}{|c|}{ DEFECTO DE LA CICATRIZ } & \multirow[b]{2}{*}{$\begin{array}{l}\text { VALOR } \\
\text { DE P }\end{array}$} \\
\hline & $S i(n=91)$ & No $(n=35)$ & \\
\hline Edad madre (años)* & $32(27-38)$ & $32(25-35)$ & 0.223 \\
\hline $\operatorname{IMC}\left(\mathrm{kg} / \mathrm{m}^{2}\right)^{*}$ & $25.1(23.8-27.1)$ & $26.0(23.2-29.0)$ & 0.985 \\
\hline $\begin{array}{l}\text { Tiempo cesárea y ecografía } \\
\text { (años)* }\end{array}$ & $5(2-10)$ & $5(2-8)$ & 0.782 \\
\hline \multicolumn{4}{|l|}{ Gestaciones } \\
\hline 1 & $17(18.7 \%)$ & $11(31.4 \%)$ & 0.298 \\
\hline 2-4 & $66(72.5 \%)$ & $21(60.0 \%)$ & \\
\hline 5-7 & $8(8.8 \%)$ & $3(8.6 \%)$ & \\
\hline Embarazo gemelar & $2(2.2 \%)$ & - & 0.377 \\
\hline Dos o más cesáreas & $48(52.8 \%)$ & $11(31.4 \%)$ & 0.032 \\
\hline Cesárea electiva & $65(71.4 \%)$ & $12(34.3 \%)$ & $<0.001$ \\
\hline
\end{tabular}




\begin{tabular}{|c|c|c|c|}
\hline $\begin{array}{l}\text { Edad gestacional cesárea } \\
\text { (semanas)* }\end{array}$ & $39(38-39)$ & $39(36-40)$ & 0.550 \\
\hline Útero en AVF & $20(22.0 \%)$ & $4(11.4 \%)$ & 0.177 \\
\hline Más de una cicatriz & $10(11.0 \%)$ & - & 0.041 \\
\hline Distancia cicatriz OCI $(\mathrm{mm}) *$ & $5.0(3.7-7.0)$ & $7.0(4.9-9.1)$ & 0.002 \\
\hline Longitud cicatriz $(\mathrm{mm})^{*}$ & $5.9(4.5-8.0)$ & $5.9(4.4-7.1)$ & 0.549 \\
\hline Amplitud cicatriz $(\mathbf{m m})^{*}$ & $5.2(3.1-7.7)$ & $3.3(2.3-5.3)$ & 0.008 \\
\hline Profundidad cicatriz $(\mathbf{m m}) *$ & $4.9(3.6-7.7)$ & $5.0(2.9-6.2)$ & 0.313 \\
\hline \multicolumn{4}{|l|}{ Miometrio residual } \\
\hline Absoluto (mm)* & $3.8(2.4-5.2)$ & $4.8(4.0-6.0)$ & 0.004 \\
\hline Relativo $(\%)^{*}$ & $42.6(28.8-55.9)$ & $50.7(41.2-61.2)$ & 0.010 \\
\hline Dispareunia & $28(30.8 \%)$ & $12(34.3 \%)$ & 0.704 \\
\hline Dolor pélvico crónico & $44(48.4 \%)$ & $17(48.6 \%)$ & 0.566 \\
\hline Dismenorrea & $52(57.1 \%)$ & $22(62.9 \%)$ & 0.675 \\
\hline Ciclos irregulares & $41(45.1 \%)$ & $13(37.1 \%)$ & 0.421 \\
\hline Aumento volumen menstrual & $43(47.3 \%)$ & $14(40.0 \%)$ & 0.464 \\
\hline $\begin{array}{l}\text { Aumento de la frecuencia } \\
\text { menstrual }\end{array}$ & $28(30.8 \%)$ & $8(22.9 \%)$ & 0.379 \\
\hline \multicolumn{4}{|l|}{ Duración menstrual } \\
\hline Similar & $47(51.6 \%)$ & $21(60.0 \%)$ & \\
\hline Mayor & $27(29.7 \%)$ & $9(25.7 \%)$ & \\
\hline Sangrado intermenstrual & $6(6.6 \%)$ & $4(11.4 \%)$ & 0.368 \\
\hline Infertilidad & $4(4.4 \%)$ & $3(8.6 \%)$ & 0.359 \\
\hline Estadio cicatriz & {$[\mathrm{n}=98]$} & {$[\mathrm{n}=37]$} & \\
\hline Leve & $35(35.7 \%)$ & $22(59.5 \%)$ & 0.014 \\
\hline Moderado & $52(53.1 \%)$ & $15(40.5 \%)$ & \\
\hline Severo & $11(11.2 \%)$ & - & \\
\hline
\end{tabular}

\section{DISCUSIÓN}

La cesárea es uno de los procedimientos quirúrgicos más comunes que se llevan a cabo en todo el mundo y es la cirugía mayormente realizada por los ginecólogos (8). Con el aumento de la tasa de cesáreas, más mujeres experimentan efectos a largo plazo, debido a que más de la mitad desarrollan un defecto en la cicatriz después de una cesárea. Estos defectos están relacionados con sangrado anormal, dismenorrea, dolor pélvico crónico y pueden estar asociados con subfertilidad y problemas obstétricos (9). 
En el presente estudio de la evaluación ecográfica de la cicatriz posterior a cesárea segmentaria en 140 mujeres no embarazadas, se encontró una prevalencia de cicatriz visible del $90 \%$, dato similar obtenido por Osser (5). Esto confirma que la ecografía transvaginal continua siendo un método útil y con alta precisión para la evaluación de la cicatriz uterina.

La prevalencia de presencia de defecto fue de $73,1 \%$, dato superior a los reportados en diferentes estudios que evidencian una prevalencia entre 24-70\%, como M. Pomorski que reporta una prevalencia de 67,1\% (9). La prevalencia de la posición del útero en AVF fue de $78,5 \%$, datos similares a los expuestos por O. Vikhareva (80\%) (5) y por M. Pomorski (69\%) en otro de sus estudios (10).

Teniendo en cuenta que el valor del miometrio residual y el porcentaje del miometrio residual son las variables que mejor predicen la indemnidad de las cicatrices uterinas, encontramos que en nuestro estudio, el $87,5 \%$ de la población presentaban un miometrio residual mayor de 2,2 mm y una media del porcentaje de miometrio residual de $45,5 \%$, datos que se correlacionan con los reportados por diferentes autores (11).

Adicionalmente, según la formas que presentaban las cicatrices, en nuestro estudio, la forma triangular y circular tuvieron una mayor prevalencia (36 y 32\% respectivamente) en relación a las demás formas descritas, lo cual es comparable con lo encontrado por Bij De Vaate et al, con una prevalencia del 50 y $37 \%$ para estas mismas formas del defecto respectivamente (12).

No se evidenció relación estadísticamente significativa entre la presencia de defectos en la cicatriz y la presencia de síntomas ginecológicos expuestos por la paciente $(\mathrm{P}>0,05)$, igualmente que con la posición del útero ni las características demográficas $(\mathrm{P}>0,05)$.

Identificamos que nuestro estudio tuvo utilidad al encontrar asociación estadística entre la cesárea electiva y la presencia de defectos en la cicatriz $(\mathrm{P}<0,001)$ contrario a lo expuesto por Tower, Hayakawa y Osser que reportan asociación entre defectos en la cicatriz y cesárea de urgencia $(13,14,15)$. Esta asociación encontrada en nuestro estudio puede deberse a que existe un adelgazamiento del segmento uterino en los embarazos a término, además que incluimos pacientes con más de una cesárea, condiciones que son reconocidas como factores de riesgo para formación de defectos. 
El presente estudio tiene varias fortalezas: resaltamos que no existen estudios similares en nuestro país, además el diseño del estudio ya que fue un estudio transversal. La técnica para la evaluación ecográfica de la cicatriz se realizó según los estándares establecidos en la literatura y exclusivamente por ecografía transvaginal utilizando un equipo de alta resolución, así como la realización de la ecografía por un sólo ginecólogo con experiencia. Adicionalmente el tiempo transcurrido entre la realización de la cesárea y de la ecografía era mayor a un año en todas las pacientes, lo que nos asegura que la cicatrización uterina estuviera completa.

Dentro de las debilidades del estudio se encuentra algunas de las variables obtenidas estaban supeditada a la memoria de las pacientes como por ejemplo el peso del recién nacido, la edad gestacional al momento de la cesárea y la indicación de la cesárea en las que tuvieron cesárea de urgencia.

\section{CONCLUSIONES}

Podemos concluir que nuestros resultados, en relación a la evaluación ecográfica de la cicatriz uterina y a la identificación de defectos, son similares a los reportados en la literatura. El miometrio residual y el porcentaje de miometrio residual se mantienen como factores importantes que sirven para determinar la cicatrización de una cesárea, resaltando que en nuestros resultados la distancia de la cicatriz al OCI es un factor protector para la formación de defectos así como la cesárea electiva también tiene asociación con las cicatrices anómalas. A pesar de no encontrar relación estadística con los síntomas ginecológicos, recomendamos incluir dentro de la evaluación ecográfica ginecológica, las medidas y características de la cicatriz en aquellas pacientes con sintomatología y antecedente de cesárea. 


\section{REFERENCIAS}

1. Beltrán AP, et al. Rates of caesarean section: analysis of global, regional and national estimates. Paediatr Perinat Epidemiol. 2007;(21):98-113.

2. Abalos E. Técnicas y materiales alternativos para cesárea: Comentario de la BSR (última revisión: 1 de abril de 2009). La Biblioteca de Salud Reproductiva de la OMS; Ginebra: Organización Mundial de la Salud. Disponible en: https://extranet.who.int/rhl/es/topics/pregnancyandchildbirth/careduringchildbirth/caes arean-section-4.

3. DANE Colombia. Nacimientos por tipo de parto según departamento de ocurrencia y sitio del parto. Disponible en: http://www.dane.gov.co/index.php/estadisticas-portema/salud/nacimientos-y-defunciones/nacimientos/nacimientos-2017.

4. Kok N, Wiersma IC, Opmeer BC, De Graaf IM, Mol BW, Pajkrt E. Sonographic measurement of lower uterine segment thickness to predict uterine rupture during a trial of labor in women with previous Cesarean section: a meta-analysis. Ultrasound Obstet Gynecol. 2013;(42):132-139.

5. Vikhareva Osser O, Jokubkiene L, Valentin L. High prevalence of defects in cesarean section scars at transvaginal ultrasound examination. Ultrasound Obstet Gynecol. 2009; (34): 90-97.

6. Jastrow N, Antonelli E, Robyr R, Irion O, Boulvain M. Inter and intraobserver variability in sonographic measurement of the lower uterine segment after a previous Cesarean section. Ultrasound Obstet Gynecol. 2006; (27):420-424.

7. Vervoort AJ, Uittenbogaard LB, Hehenkamp WJ, Brölmann HA, Mol BW, Huirne JA. Why do niches develop in caesarean uterine scar? Hypotheses on the aetiology of niche development. Hum Reprod. 2015;30(12):2695-2702.

8. van der Voet LF, Bij de Vaate AM, Heymans MW, Brölmann HA, Veersema S, Huirme JA. Prognostic factors for niche development in the uterine caesarean section scar. Eur J Obstet Gynecol Reprod Biol. 2017;(213):31-32.

9. Pomorski M, Fuchs T, Rosner-Tenerowicz A, Zimmer M. Standardized ultrasonographic approach for the assessment of risk factors of incomplete healing of the cesarean section scar in the uterus. Eur J Obstet Gynecol Reprod Biol. 2016;(205):141-145. 
10. Pomorski M, Fuchs T, Rosner-Tenerowicz A, Zimmer M. Morphology of the cesarean section scar in the non-pregnant uterus after one elective cesarean section. Ginekol Pol. 2017;88(4):174-179.

11. Pomorski M, Fuchs T, Zimmer. Prediction of uterine dehiscence using ultrasonographic parameters of cesarean section scar in the nonpregnant uterus: a prospective observational study. BMC Pregnancy and Childbirth. 2014;(14):365.

12. Bij de Vaate AM et al. Prevalence, potential risk factors for development and symptoms related to the presence of uterine niches following Cesarean section: systematic review. Ultrasound Obstet Gynecol. 2014;(43):372-382.

13. Tower AM, Frishman GN. Cesarean scar defects: an underrecognized cause of abnormal uterine bleeding and other gynecologic complications. Journal Minim Invasive Gynecol. 2013;(20):562-572.

14. Hayakawa A, et al. Method for myometrium closure and other factors impacting effects on cesarean section scar of the uterine segment detected by the ultrasonography. Acta Obstet Gynecol Scand. 2006;85(4):429-434.

15. Vikhareva Osser O, Valentin L. Risk factors for incomplete healing of the uterine incision after caesarean section. BJOG. 2010;(117):1119-1126. 


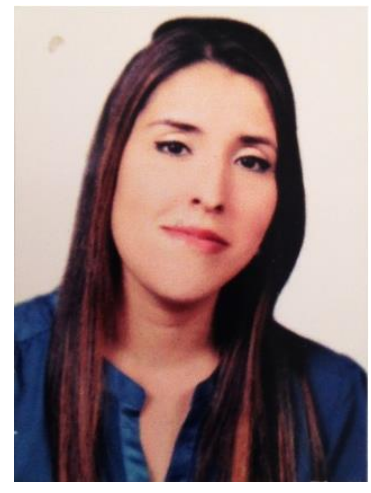

NATALY PAOLA NEGRETTE LOPERA

Es especialista en Ginecología y Obstetricia de la Universidad Industrial de Santander, Bucaramanga, Colombia. Médica Cirujana egresada en el año 2011 de la Universidad del Norte en Barranquilla, Colombia, con promedio académico de estudiante distinguido. Siempre interesada en la salud de la mujer en sus diferentes etapas de la vida. Autora principal del estudio: "Características ecográficas de la cicatriz postcesárea en el segmento uterino inferior en pacientes no gestantes", primer artículo de investigación realizado que sirvió como tesis para la obtención de su título de especialista.

Is a specialist in Gynecology and Obstetrics at the Universidad Industrial de Santander, Bucaramanga, Colombia. Medical surgeon graduated in 2011 from the Universidad del Norte in Barranquilla, Colombia, with an academic average of distinguished student. Always interested in the health of women in their different stages of life. Main author of the study: "Ecographic characteristics of post-cesarean scar in the lower uterine segment in nonpregnant patients", first article of research carried out that served as thesis for obtaining its specialist title. 